\title{
Changes in dermatological complaints among healthcare professionals during the COVID-19 outbreak in Turkey
}

\author{
Nurcan Metin ${ }^{1 凶}$, Çağrı Turan ${ }^{1}$, Zeynep Utlu \\ ${ }^{1}$ Department of Dermatology and Venereology, University of Health Sciences, Erzurum Regional Training and Research Hospital, Erzurum, Turkey.
}

\begin{abstract}
Introduction: This questionnaire study evaluates dermatological complaints that may arise due to hygiene measures and anxiety among healthcare professionals (HCPs) during the COVID-19 outbreak.

Methods: A total of 526 volunteers, consisting of doctors and nurses, participated. Demographic features, personal hygiene behavior, personal protective equipment (PPE) use, Hospital Anxiety and Depression Scale-Anxiety (HADS-A) parameters, and symptoms of various dermatological diseases (xerosis, eczema, acne, hair loss, palmar hyperhidrosis, xeromycteria, urticaria, aphthous stomatitis, and seborrheic dermatitis) were investigated.

Results: Although the frequency and severity of many dermatological complaints increased during the pandemic period, the most frequent increase was observed in the frequency of complaints suggesting xerosis and eczema. We found that complaints suggesting xerosis and eczema were seen on the hands 2.44 and 3.57 times, respectively, as a result of washing hands 10 times/day, and that handwashing times of 10 seconds or more significantly increased the risk of eczema (5.44 times). Another remarkable result was a fivefold increase in acne complaints among those using any mask. The severity of all complaints except hair loss and seborrheic dermatitis correlated significantly with HADS-A.
\end{abstract}

Conclusions: Our study showed that the frequency and severity of some dermatological complaints increased in HCPs.

Keywords: COVID-19, pandemic, HADS-A, dermatological complaints

Received: 5 May 2020 | Returned for modification: 11 June 2020 | Accepted: 30 June 2020

\section{Introduction}

Pneumonia cases caused by a newly identified coronavirus arose in Wuhan, China in December 2019. The World Health Organization (WHO) named this virus the 2019 new coronavirus (2019$\mathrm{nCoV}$ ) and the disease coronavirus disease 2019 (COVID-19) on January 12th, 2020. Available data indicated that wild animals sold at the Huanan Seafood Wholesale Market were the source of this disease (1). COVID-19, which started in China, affected many countries in a short time and spread all over the world. The first case in Turkey was seen on March 11th, 2020, the day the WHO declared the pandemic (2). Transmission mainly takes place through large droplets that occur during coughing and sneezing by symptomatic patients. Touching the nose, mouth, and eye mucosa after contact with contaminated surfaces may also pose a risk of transmission (3). The incubation period ranges from 2 to 14 days. This disease has been observed in all age groups. Men have been more affected (4). The clinical features of COVID-19 range from asymptomatic status to pneumonia, acute respiratory distress syndrome, and multi-organ failure. The most common symptoms reported are fever, dry cough, chest pain, and shortness of breath $(5,6)$. In most cases, COVID-19 has a mild course, and patients recover within 2 to 3 weeks. This disease may have a more severe course in elderly patients and patients with underlying comorbidity. Therefore, these patient groups can be defined as high-risk groups for COVID-19. It was reported that, among COVID-19 patients that died, $42.2 \%$ were 80 to 89 years old, $32.4 \%$ were 70 to 79 years old, and $8.4 \%$ were 60 to 69 years old ( 7 ).

Healthcare professionals (HCPs) struggling with COVID-19 in the front lines are in the high-risk group for transmission. A recent article reported that 3,300 HCPs were infected and at least 22 (about $0.7 \%$ ) of them died in early March, according to data from China's National Health Commission, and also that $20 \%$ of healthcare workers in Italy were infected (7). Therefore, especially HCPs are aware that they must protect themselves through methods such as effective hand hygiene and the use of various forms of personal protective equipment (PPE). Increased use of masks and gloves as part of personal hygiene measures can cause an increase in the frequency and severity of various dermatoses (8). Furthermore, increased anxiety levels of HCPs in this pandemic process may also induce some psychodermatoses.

We primarily aimed to evaluate the change in the frequency and severity of various symptoms associated with dermatoses in doctors and nurses during the COVID-19 outbreak. Our secondary goal was to investigate the factors that may be related to the dermatoses that occur.

\section{Method}

From April 5th to 12th, 2020, between the 25th and 32nd days after the first COVID-19 case in Turkey, a self-administered online questionnaire (SurveyMonkey ${ }^{\circledR)}$ consisting of 90 questions was conducted for doctors and nurses that voluntarily participated in the study. We aimed to reduce the risk of bias in the questions posed to the participants by carrying out this retrospective study in the early phase of the COVID-19 outbreak. The first part of the questionnaire examined subjects such as demographic characteristics, occupational and institutional information, medical history, 
hygiene, and personal care behaviors before the COVID-19 outbreak and in the previous week, and PPE use during the previous month. The second part asked about symptoms pointing to various diseases such as xerosis cutis, xeromycteria, eczema, acne, hair loss, palmar hyperhidrosis (PH), aphthous stomatitis (AS), urticaria (acute or chronic), and seborrheic or seborrheic-like dermatitis (SD). Volunteers were asked if they had had these symptoms in the previous week and in the first week just before the COVID-19 outbreak. In addition, they were asked to evaluate the severity of their complaints on a scale of o to 10 using the Visual Analogue Scale (VAS). PPE-related symptoms such as erythema or erosion were also asked about. The current anxiety levels of the participants were determined using the Hospital Anxiety and Depression Scale-Anxiety (HADS-A) with a score of o to 21 points, which consists of seven questions and was validated in Turkish by Aydemir et al. (9). The participants' data were documented and anonymized. The interpretation of the HADS-A scores was based on the recommended cut-off values in the literature: 0 to 7 points indicates that there is no anxiety, 8 to 10 points indicates mild anxiety, 11 to 14 points indicates moderate anxiety, and 12 to 21 points indicates severe anxiety $(9,10)$.

Conducting this study for HCPs made it possible to make the survey questions clearer, and this made the results more reliable. We tried to ask about the symptoms in terms that were as short and clear as possible. Symptoms indicating diseases are indicated in parentheses (such as acne, PH; Table S1). Common diseases, such as rosacea and atopic dermatitis, which are more difficult to distinguish from other diseases on the list, are excluded. Although hair loss as a symptom was evaluated, alopecia areata was excluded. No distinction was made between telogen effluvium (TE) and androgenetic alopecia (AGA), which are the other most likely diagnoses for women and men in hair loss, respectively. Hair loss was evaluated separately by sex due to the differences in its diagnostic distribution in women and men. The inclusion criteria in this questionnaire were determined as being a doctor or nurse and working actively in any health institution in Turkey. HCPs that were on leave for any reason or retired, and high-school graduate nurses were excluded.

This study was approved by the Ethics Committee of Erzurum Regional Training and Research Hospital, Erzurum, Turkey (decision no. 2020/08-91) and was conducted in line with the latest version of the Helsinki Declaration and the Guidelines for Good Clinical Practice.

Available data were collected from 526 volunteers. After checking the normality distribution of scale variables with the Kolmogorov-Smirnov test, independent samples were compared with appropriate significance tests (Mann-Whitney $U$ and Kruskal-Wallis $H$ ) and the Wilcoxon test was used for dependent samples. For categorical variables, Pearson's chi-square and Fisher's exact tests were used, which were appropriate. We also performed a univariate logistic regression for complaints indicating diseases (dependent variable), establishing the crucial impacting factors such as age, sex, HADS-A, and etiological factors (independent variables) and their odds ratios (OR). The associations between the severity of complaints and HADS-A scores were analyzed using Spearman's partial correlation analysis. A level of 5\% type-1 error was considered statistically significant. All statistical analyses were performed using IBM SPSS Statistics 21.0 and MS-Excel 2010.

\section{Results}

\section{Demographic data}

This questionnaire study involved 526 volunteers; 353 of them (143 men and 210 women; 40.5\% vs. 59.5\%) were doctors and 173 (19 men and 154 women; $11.0 \%$ vs. $89.0 \%$ ) nurses. The proportion of women among all the participants was $69.2 \%$. The ages of the participants ranged from 24 to 57 years (mean \pm standard deviation $(S D)=34 \pm 7$ years) for doctors and 22 to 54 years ( $34 \pm 14$ years) for nurses. Although there was a statistically significant difference in terms of the sex distribution between doctors and nurses, the age distributions were similar ( $p<0.001$ and $p=0.252$, respectively).

This study was performed during the period when COVID-19 was seen across the country. At least three HCPs from 60 (74.1\%) of 81 provinces participated in the study. There were $163(31.5 \%)$, $62(12.0 \%), 50$ (9.7\%), and 49 (9.5\%) participants from Erzurum, Elazığ, Ankara, and Istanbul, respectively. HCPs in these four provinces constituted $62.7 \%$ of the participants. Three hundred fifteen of the volunteers $(60.9 \%)$ resided in a province with a population of less than 1 million, and 153 (29.6\%) resided in a province with a population of 1 to 6 million. The population of Istanbul, which constitutes the third group alone, is more than 15 million. There was no significant difference between the population of the provinces and the HADS-A scores of the participants ( $p=0.548$ ).

Doctors from 38 different specialties, including internal, surgical, and basic sciences, participated in this study. Of these, 252 (74.2\%) were specialist physicians and 91 (25.8\%) were general practitioners. They worked in primary $(72,20.4 \%)$, secondary $(88$, $24.9 \%$ ), and tertiary $(170,48.2 \%)$ healthcare departments, and private hospitals or clinics $(23,6.5 \%)$. Among the participants were 311 (59.1\%) HCPs working in the quarantine hospital. HADSA scores were not significantly associated with working in healthcare departments or quarantine hospitals ( $p=0.879$ and $p=0.939$, respectively). Physicians were divided into three groups according to their assignment related to COVID-19, not according to their specialties. Accordingly, 114 (33.2\%) physicians were assigned to the COVID-19 outpatient clinics, inpatient services, or intensive care units (high risk) and 35 (10.2\%) were assigned to the emergency department (moderate risk). Among the participants, 194 (56.6\%) physicians continued to work in their outpatient clinics (low-risk group). Among the nurses, 122 (71.3\%) were not assigned to a task related to COVID-19, 14 (8.2\%) were assigned to emergency departments, and 35 (20.5\%) were assigned to COVID-19 outpatient clinics, inpatient services, or intensive care units. No significant relation was found between the risk levels of the units they work in and the HADS-A scores of doctors and nurses ( $p=$ 0.827 and $p=0.185$, respectively). The HADS-A scores of men and women were found to be similar ( $p=0.391$ and $p=0.346$, respectively). Doctors and nurses worked about 40 hours a week with flexible working hours during the COVID-19 outbreak, and there was no significant difference between them in terms of working hours $(p=0.747)$. There was no correlation between working time and anxiety levels $(r=0.03, p=0.594)$.

One hundred of the participants (19.4\%) had at least one chronic disease. Sixteen (3\%) had diabetes mellitus (DM) and $21(4 \%)$ had hypertension (HT). In the 1st month of the onset of the COVID-19 outbreak, an increase of $30 \pm 28 \%$ was detected in the severity of complaints of 16 participants (3\%) with any chronic skin disease. 


\section{PPE use and hygiene measures}

It was determined that $96.7 \%$ of the volunteers used masks in the hospital and $75.3 \%$ of them in their daily lives. Gloves were not worn by $9.9 \%, 21.9 \%$ wore them for less than 1 hour, and $22.2 \%$ for more than 8 hours. One hundred forty-five of the 517 participants (28\%) described complaints in at least one area of the face in the form of redness or erosion around the nose, forehead, eyes, or ears related to PPE use, such as masks, goggles, and face shields. Complaints related to the use of masks or glasses were described by 82 participants (15.9\%) on the nasal bridge and 77 $(14.9 \%)$ around the ear. In addition, $51.2 \%$ of the participants with complaints on the nasal bridge and $58.4 \%$ of the participants with complaints around the ear had at least one other area of involvement. Forty-one participants (7.9\%) described complaints related to the use of goggles and face shields in their periocular areas and 29 participants (5.6\%) on their foreheads.

Liquid soap was used during hand washing by $87.7 \%$ of the participants, and $12.3 \%$ used bar soap. The rate of those that used hand sanitizer among participants was $77.4 \%$. Soap was used when washing their faces by $74.8 \%$ of the participants, and $25.2 \%$ used tap water only. Changes in the frequency of personal care and hygiene before the COVID-19 outbreak and 1 month after the onset of the outbreak are presented in Table 1. Accordingly, the frequency and duration of hygiene behavior and the need to use moisturizers increased significantly within 1 month after the on- set of the COVID-19 outbreak. The frequency of handwashing in women was found to be significantly higher both before and after the onset of the COVID-19 outbreak $(p<0.001)$. However, other hygiene behavior did not differ by sex both before and after the onset of the COVID-19 outbreak ( $p>0.05)$.

\section{Dermatological complaints}

Changes in the frequency and severity of various dermatological complaints in HCPs before the COVID-19 outbreak and 1 month after the onset of the outbreak are presented in Figure 1 and Table 2. As seen in Figure 1, there was an increase in the frequency of all complaints except hair loss. The frequency of complaints suggesting hair loss and acute or chronic urticaria differed in both sexes (Table 2). It was determined that there was an increase only in women in the severity of complaints suggesting diseases such as xerosis or eczema on hands, face, and body, hair loss, PH, acute or chronic urticaria, and AS. There was no significant increase in acne severity in both sexes. It was noted that the frequency and severity of all other complaints in Table 2 increased significantly in both sexes. Apart from these, 24 participants (4.6\%) stated that they had shingles before and only one person had shingles recently. Five participants stated that they had alopecia areata before the COVID-19 outbreak, but no participants had recently developed alopecia areata.

The relationship between the frequencies of various dermato-

Table 1 | Changes in frequencies of hygiene and personal care after the COVID-19 outbreak compared to before.

\begin{tabular}{|c|c|c|c|c|c|c|c|}
\hline & & \multirow{2}{*}{ Before COVID-19 } & \multirow{2}{*}{ After 1 month } & \multirow{2}{*}{$Z$} & \multirow{2}{*}{$p$-valuet } & \multicolumn{2}{|c|}{ Correlation with HADS-A‡ } \\
\hline & & & & & & $r$ & $p$-value \\
\hline \multirow[t]{5}{*}{ Handwashing (times/day, $n=521$ ) } & $1-4$ & $69(13.2 \%)$ & $2(0.4 \%)$ & \multirow{5}{*}{-19.2} & \multirow{5}{*}{$<0.001$} & \multirow{5}{*}{0.109} & \multirow{5}{*}{0.015} \\
\hline & $5-9$ & $197(37.8 \%)$ & $25(4.8 \%)$ & & & & \\
\hline & $10-14$ & $159(30.5 \%)$ & $88(16.9 \%)$ & & & & \\
\hline & $15-19$ & $54(10.4 \%)$ & $118(22.6 \%)$ & & & & \\
\hline & $>20$ & $42(8.1 \%)$ & $288(55.3 \%)$ & & & & \\
\hline \multirow[t]{4}{*}{ Handwashing time per event (seconds, $n=523$ ) } & $0-9$ & $180(34.4 \%)$ & $9(1.7 \%)$ & \multirow{4}{*}{-19.3} & \multirow{4}{*}{$<0.001$} & \multirow{4}{*}{0.066} & \multirow{4}{*}{0.141} \\
\hline & $10-19$ & $274(52.4 \%)$ & $111(21.2 \%)$ & & & & \\
\hline & $20-29$ & $58(11.1 \%)$ & $313(59.8 \%)$ & & & & \\
\hline & $>30$ & $11(2.1 \%)$ & $90(17.2 \%)$ & & & & \\
\hline Face washing (times/day, $n=513$ ) & & $3(I Q R=3)$ & $5(I Q R=4)$ & -16.4 & $<0.001$ & 0.062 & 0.167 \\
\hline Shower frequency (times/week, $n=522$ ) & & $3(I Q R=2)$ & $5(\mathrm{IQR}=3)$ & -17.4 & $<0.001$ & 0.173 & $>0.001$ \\
\hline Need moisturizer (times/day, $n=525$ ) & & $1(\mathrm{IQR}=2)$ & $3(\mathrm{IQR}=4)$ & -15.6 & $<0.001$ & 0.083 & 0.065 \\
\hline
\end{tabular}

HADS-A = Hospital Anxiety and Depression Scale-Anxiety (after COVID-19 outbreak), IQR = interquartile range. Data are expressed as number of patients (\%) or median (IQR), whichever is appropriate. Significant values are shown in bold. WWilcoxon test used, ¥Spearman's rho partial correlation test with controlling sex and residence with a person at high risk of death from COVID-19 used by syntax.

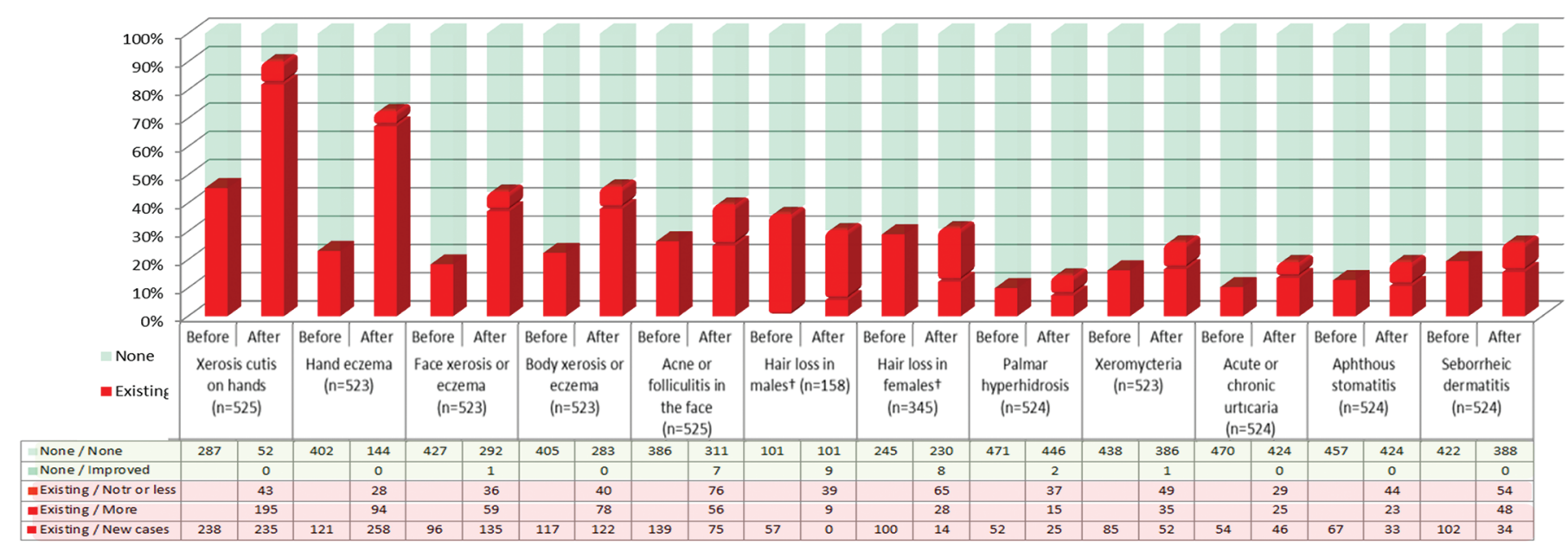

Figure 1 | Changes in frequency and severity of various dermatological complaints before COVID-19 outbreak and after 1 month. "Yes" responses in the "After" columns were divided into two parts. In terms of severity of the disease before and after, the lower part of the bar corresponds to new cases and those with an increase, and the upper part to those with no change or a decrease. †Volunteers with possible telogen effluvium or androgenic alopecia were evaluated by excluding alopecia areata. 
logical complaints before the COVID-19 outbreak and 1 month after the onset of the outbreak and other various parameters including HADS-A are presented in Table S2. Data with a $p$-value below 0.1 were evaluated by binary logistic regression analysis and are presented in Table 3.

The mean HADS-A score of the participants was 8.1 \pm 3.5 , ranging from o to 16 points. No significant correlation was found between age and HADS-A score $(r<0.01, p=0.974)$. The mean HADS-A score was $8.4 \pm 3.2$ in women and $6.9 \pm 3.7$ in men, and this difference was statistically significant $(p<0.001)$. The frequency of residence with a person at high risk of death from COVID-19 was found to be significantly higher in women $(25.3 \%)$ than men $(14.9 \%, p=$ o.008). The HADS-A score was $8.6 \pm 3.6$ in those residing with a person at high risk of death from COVID-19, and $7.8 \pm 3.5$ in those that did not, and the difference between these two groups was statistically significant $(p=0.037)$. The HADS-A scores of those residing with a person at high risk of death from COVID-19 and those that did not were found to be similar in women but were found to be statistically significantly different in men ( $p=0.471$ and $p=$ 0.049 , respectively). Apart from these, no relationship was found between HADS-A score and marital status, and having children or having DM, HT, or any chronic disease ( $p>0.05)$. Therefore, while investigating the relationship between dermatological complaints and HADS-A scores, analyses were carried out by controlling for the sex and status of residence with a person at high risk of death from COVID-19. The correlation of severity of various dermatological diseases with anxiety levels after the COVID-19 outbreak is presented in Table 4. A weak but significant linear correlation was found between the severity of all complaints and HADS-A scores except for complaints suggesting hair loss and SD.

\section{Discussion}

The WHO and Centers for Disease Control and Prevention (CDC) recommend PPE use and hand hygiene for HCPs to prevent virus transmission in the fight against COVID-19 $(11,12)$. A current meta-analysis concluded that a $24 \%$ reduction in viral transmission can be achieved by just handwashing (13). The CDC recommends handwashing for at least 20 seconds during the pandemic, especially after risky contact (12). PPE such as hand sanitizer, gloves, face masks, glasses, face shields, hair caps, and disposable gowns are being used more than ever in hospitals during the pandemic period. In our study, significant increases in the frequency of personal care and hygiene behavior have been confirmed.

High anxiety levels, exaggerated or irrational protection behaviors, and some measures against COVID-19 may trigger or aggravate the emergence of various dermatological complaints. In this study, after the onset of the COVID-19 outbreak, various complaints that could be triggered by protection measures or stress in HCPs and easily questioned in volunteers were discussed. Although the symptoms questioned indicate certain dermatological diseases, the presence of these symptoms does not confirm the diagnosis. Significant increases were found in the frequency and severity of most of the diseases examined after the onset of the COVID-19 outbreak (Fig. 1, Table 2). Previous studies revealed that hand eczema was quite common in HCPs $(14,15)$. Recently, it was report-

Table 2 | Changes in frequency and severity of various dermatological complaints by sex before COVID-19 outbreak and after 1 month.

\begin{tabular}{|c|c|c|c|c|c|c|c|c|c|c|}
\hline \multirow{2}{*}{ Diseases } & \multirow{2}{*}{ Sex } & \multicolumn{4}{|c|}{ Change in disease frequency* } & \multicolumn{5}{|c|}{ Change in disease severity (VAS)** } \\
\hline & & $n$ & Before & After & $p$-valuet & $n$ & Before & After & $Z$ & $p$-value $\neq$ \\
\hline \multirow[t]{3}{*}{ Xerosis cutis on hands } & M & 161 & $48(29.8 \%)$ & $120(74.5 \%)$ & $<0.001$ & 46 & $4(2)$ & $6(3)$ & -4.1 & $<0.001$ \\
\hline & $\mathrm{F}$ & 364 & $190(52.2 \%)$ & $353(97.0 \%)$ & $<0.001$ & 188 & $4(3)$ & $8(3)$ & -7.9 & $<0.001$ \\
\hline & Total & 525 & $238(45.3 \%)$ & $473(90.1 \%)$ & $<0.001$ & 234 & $4(3)$ & $7(3)$ & -12.1 & $<0.001$ \\
\hline \multirow[t]{3}{*}{ Hand eczema } & $M$ & 161 & $31(19.3 \%)$ & $90(55.9 \%)$ & $<0.001$ & 31 & $5(3)$ & $6(4)$ & -1.9 & 0.056 \\
\hline & $\mathrm{F}$ & 362 & $90(24.9 \%)$ & $289(79.8 \%)$ & $<0.001$ & 90 & $4.5(3)$ & $8(3)$ & -6.9 & $<0.001$ \\
\hline & Total & 523 & $121(23.1 \%)$ & $379(72.5 \%)$ & $<0.001$ & 121 & $5(3)$ & $7(4)$ & -7.1 & $<0.001$ \\
\hline \multirow[t]{3}{*}{ Facial xerosis or eczema } & $M$ & 161 & $16(9.9 \%)$ & $41(25.5 \%)$ & $<0.001$ & 14 & $4(5)$ & $6(5)$ & -0.5 & 0.622 \\
\hline & $\mathrm{F}$ & 362 & $80(22.1 \%)$ & $189(52.2 \%)$ & $<0.001$ & 81 & $3(4)$ & $5(5)$ & -3.8 & $<0.001$ \\
\hline & Total & 523 & $96(18.4 \%)$ & $230(44.9 \%)$ & $<0.001$ & 95 & $4(4)$ & $5(5)$ & -3.7 & $<0.001$ \\
\hline \multirow[t]{3}{*}{ Body xerosis or eczema } & M & 161 & $30(18.6 \%)$ & $51(31.7 \%)$ & $<0.001$ & 29 & $5(4)$ & $5(6)$ & -0.3 & 0.795 \\
\hline & $\mathrm{F}$ & 362 & 87 (24.1\%) & $188(52.1)$ & $<0.001$ & 87 & $4(3)$ & $6(5)$ & -4.0 & $<0.001$ \\
\hline & Total & 523 & $117(22.4 \%)$ & $239(45.8 \%)$ & $<0.001$ & 116 & $4(4)$ & $6(6)$ & -3.6 & $<0.001$ \\
\hline \multirow{3}{*}{$\begin{array}{l}\text { Acne or folliculitis on } \\
\text { face }\end{array}$} & $M$ & 161 & $27(16.8 \%)$ & $36(22.4 \%)$ & 0.012 & 25 & $3(2)$ & $2(5)$ & -1.7 & 0.087 \\
\hline & $\mathrm{F}$ & 364 & $112(30.8 \%)$ & $171(47.0 \%)$ & $<0.001$ & 105 & $3(2)$ & $3(5)$ & -0.8 & 0.445 \\
\hline & Total & 525 & $139(26.5 \%)$ & 207 (39.4\%) & $<0.001$ & 130 & $3(2)$ & $3(5)$ & -1.4 & 0.175 \\
\hline \multirow[t]{3}{*}{ Hair loss§ } & $M$ & 158 & $57(36.1 \%)$ & $48(30.4 \%)$ & 0.004 & 45 & $5(4)$ & $5(4)$ & -0.4 & 0.670 \\
\hline & $F$ & 345 & $100(29.0 \%)$ & $107(31.0 \%)$ & 0.210 & 88 & $4(3)$ & $5(3)$ & -4.0 & $<0.001$ \\
\hline & Total & 503 & $157(31.2 \%)$ & $155(30.8 \%)$ & 0.860 & 133 & $4(3$ & $5(4)$ & -3.6 & $<0.001$ \\
\hline \multirow[t]{3}{*}{ Palmar hyperhidrosis } & $M$ & 161 & $21(13.0 \%)$ & $26(16.1 \%)$ & 0.125 & 20 & $3(4)$ & $4(3)$ & -1.5 & 0.123 \\
\hline & $F$ & 363 & 31 (8.6\%) & $49(13.5 \%)$ & $<0.001$ & 30 & $4(2)$ & $4(2)$ & -2.2 & 0.031 \\
\hline & Total & 524 & 52 (9.9\%) & $75(14.3 \%)$ & $<0.001$ & 50 & $3.5(2)$ & $4(2)$ & -2.6 & 0.010 \\
\hline \multirow[t]{3}{*}{ Xeromycteria } & $M$ & 161 & $28(17.4 \%)$ & $35(21.7 \%)$ & 0.039 & 25 & $3(3)$ & $4(4)$ & -2.6 & 0.010 \\
\hline & $F$ & 362 & $57(15.7 \%)$ & $101(27.9)$ & $<0.001$ & 56 & $4(3)$ & $4(3)$ & -3.6 & $<0.001$ \\
\hline & Total & 523 & $85(16.3 \%)$ & $136(26.0 \%)$ & $<0.001$ & 81 & $3(3)$ & $4(3)$ & -4.4 & $<0.001$ \\
\hline \multirow[t]{3}{*}{ Acute or chronic urticaria } & M & 162 & $14(8.6 \%)$ & $19(11.7 \%)$ & 0.063 & 13 & $4(4)$ & $5(3)$ & -1.5 & 0.131 \\
\hline & $\mathrm{F}$ & 362 & $40(11.0 \%)$ & $81(22.4 \%)$ & $<0.001$ & 38 & $3(3)$ & $4(3)$ & -2.5 & 0.013 \\
\hline & Total & 524 & $54(10.3 \%)$ & $100(19.1 \%)$ & $<0.001$ & 51 & $3(3)$ & $4(3)$ & -2.9 & 0.003 \\
\hline \multirow[t]{3}{*}{ Aphthous stomatitis } & M & 161 & $19(11.8 \%)$ & $25(15.5 \%)$ & 0.031 & 18 & $1(2)$ & $1(3)$ & -1.5 & 0.141 \\
\hline & $\mathrm{F}$ & 363 & $48(13.2 \%)$ & 75 (20.7\%) & $<0.001$ & 46 & $3(3)$ & $4(2)$ & -3.7 & $<0.001$ \\
\hline & Total & 524 & $67(12.8 \%)$ & $100(19.1 \%)$ & $<0.001$ & 64 & $3(4)$ & $3(3)$ & -3.6 & $<0.001$ \\
\hline \multirow[t]{3}{*}{ Seborrheic dermatitis } & $M$ & 162 & $36(22.2 \%)$ & $46(28.4 \%)$ & 0.002 & 32 & $4(4)$ & $4.5(6)$ & -2.5 & 0.011 \\
\hline & $\mathrm{F}$ & 362 & $66(18.2 \%)$ & $90(24.9 \%)$ & $<0.001$ & 62 & $4(2)$ & $4(2)$ & -4.6 & $<0.001$ \\
\hline & Total & 524 & $102(19.5 \%)$ & $136(26.0 \%)$ & $<0.001$ & 94 & $4(2)$ & $5(3)$ & -5.2 & $<0.001$ \\
\hline
\end{tabular}

VAS $=$ visual analog scale. Significant values are shown in bold. *Data expressed as number of patients (\%), ** data expressed as a median VAS (interquartile range), §volunteers with possible telogen effluvium or androgenic alopecia evaluated by excluding alopecia areata, †McNemar test used, ¥Wilcoxon test used. 
ed that $74.5 \%$ of HCPs had irritation on the hands after the onset of the COVID-19 outbreak (16). Therefore it is not surprising that the most significant increase in frequency was especially in complaints suggesting xerosis and/or eczema on the hands, face, or body. It was remarkable that no participants reported a decrease in their complaints during the previous month. On the contrary, new cases with complaints suggesting acne, $\mathrm{PH}$, xeromycteria, $\mathrm{AS}$, and SD appeared, but many previous cases partially improved. It was noteworthy that, unlike women, for men there were no new cases of hair loss, and no remarkable complete recovery was observed in

Table 3 | Logistic regression analyses of parameters associated with frequencies of various dermatological complaints before COVID-19 outbreak and after 1 month.

\begin{tabular}{|c|c|c|c|c|c|c|c|c|}
\hline \multirow{2}{*}{ Univariate logistic regression analysis } & & \multirow{2}{*}{$\beta \mathbf{i}$} & \multirow{2}{*}{ Odds ratio } & \multicolumn{2}{|c|}{$95 \% \mathrm{Cl}$} & \multirow{2}{*}{ Wald value } & \multirow{2}{*}{$R^{2}$} & \multirow{2}{*}{$p$-value } \\
\hline & & & & Lower & Upper & & & \\
\hline \multicolumn{9}{|l|}{ Xerosis cutis on hands (dryness or fissures on hands) } \\
\hline \multirow[t]{2}{*}{ Sex: female } & Before & 0.95 & 2.59 & 1.75 & 3.85 & 22.36 & 0.044 & $<0.001$ \\
\hline & After & 2.40 & 10.96 & 5.46 & 22.01 & 45.34 & 0.109 & $<0.001$ \\
\hline \multirow[t]{2}{*}{ HADS-A score $>7$ points } & Before & - & - & - & - & - & - & - \\
\hline & After & 1.78 & 5.95 & 2.80 & 12.64 & 21.48 & 0.052 & $<0.001$ \\
\hline \multirow[t]{2}{*}{ Residence with person at high COVID-19 death risk: yes } & Before & 0.15 & 1.16 & 0.76 & 1.76 & 0.47 & 0.001 & 0.493 \\
\hline & After & 0.85 & 2.34 & 1.04 & 5.62 & 3.60 & 0.008 & 0.048 \\
\hline \multirow[t]{2}{*}{ Handwashing $\geq 10$ times/day } & Before & 0.53 & 1.70 & 1.20 & 2.40 & 8.86 & 0.017 & 0.003 \\
\hline & After & 0.89 & 2.44 & 1.34 & 4.46 & 8.45 & 0.015 & 0.004 \\
\hline \multirow[t]{2}{*}{ Hand soap preference: liquid soap } & Before & - & - & - & - & - & - & - \\
\hline & After & 0.03 & 1.03 & 0.38 & 2.78 & 0.003 & $<0.001$ & 0.953 \\
\hline Hand sanitizer use: yes & Before & - & - & - & - & - & - & - \\
\hline & After & 0.99 & 2.70 & 0.85 & 8.58 & 2.82 & 0.005 & 0.093 \\
\hline Need moisturizer $\geq 3$ times/day & Before & 0.55 & 1.73 & 1.13 & 2.64 & 6.44 & 0.012 & 0.011 \\
\hline & After & 1.91 & 6.72 & 2.82 & 16.04 & 18.44 & 0.051 & $<0.001$ \\
\hline Using gloves: yes & Before & - & - & - & - & - & - & - \\
\hline & After & 0.99 & 2.68 & 1.28 & 5.59 & 6.88 & 0.011 & 0.009 \\
\hline Wearing duration of gloves $\geq 2$ hours & Before & - & - & - & - & - & - & - \\
\hline & After & 0.79 & 2.21 & 1.22 & 4.36 & 5.26 & 0.012 & 0.022 \\
\hline Hand eczema (redness, edema, weeping, crusting, burning & , pain, o & pruritus & on hand skin) & & & & & \\
\hline Age $\leq 30$ years & Before & 0.06 & 1.06 & 0.70 & 1.61 & 0.08 & $<0.001$ & 0.779 \\
\hline & After & 0.56 & 1.76 & 1.16 & 2.67 & 7.04 & 0.014 & 0.008 \\
\hline Sex: female & Before & 0.33 & 1.39 & 0.88 & 2.20 & 2.01 & 0.004 & 0.156 \\
\hline & After & 1.14 & 3.13 & 2.09 & 4.69 & 30.81 & 0.057 & $<0.001$ \\
\hline HADS-A score $>7$ points & Before & - & - & - & - & - & - & - \\
\hline & After & 0.93 & 2.53 & 1.69 & 3.79 & 20.25 & 0.040 & $<0.001$ \\
\hline Residence with person at high COVID-19 death rish: yes & Before & -0.04 & 0.96 & 0.59 & 1.58 & 0.024 & $<0.001$ & 0.878 \\
\hline & After & 0.82 & 2.27 & 1.33 & 3.88 & 9.03 & 0.019 & 0.003 \\
\hline Handwashing $\geq 10$ times/day & Before & 0.81 & 2.24 & 1.47 & 3.42 & 14.06 & 0.027 & $<0.001$ \\
\hline & After & 1.27 & 3.57 & 1.63 & 7.83 & 10.11 & 0.019 & 0.001 \\
\hline Hand wash time per event $\geq 10$ seconds & Before & 0.16 & 1.17 & 0.76 & 1.82 & 0.52 & 0.001 & 0.470 \\
\hline & After & 1.69 & 5.44 & 1.34 & 22.03 & 5.62 & 0.012 & 0.018 \\
\hline Need moisturizer $\geq 3$ times/day & Before & 0.56 & 1.75 & 1.10 & 2.79 & 5.56 & 0.010 & 0.018 \\
\hline & After & 1.21 & 3.35 & 2.24 & 5.00 & 34.85 & 0.067 & $<0.001$ \\
\hline Wearing duration of gloves $\geq 1$ hour $¥$ & Before & - & - & - & - & - & - & - \\
\hline & After & 0.58 & 1.77 & 1.15 & 2.77 & 8.05 & 0.016 & 0.005 \\
\hline Facial xerosis or eczema (redness, dryness, fissures, weep & ing, crust & ng, burr & ng, pain, or $p$ & eritus on & ce skin) & & & \\
\hline Age $>30$ years & Before & 0.56 & 1.75 & 1.08 & 2.85 & 5.12 & 0.010 & 0.024 \\
\hline & After & -0.06 & 0.94 & 0.66 & 1.34 & 0.113 & $<0.001$ & 0.736 \\
\hline Sex: female & Before & 0.96 & 2.60 & 1.47 & 4.61 & 10.73 & 0.023 & 0.001 \\
\hline & After & 1.16 & 3.20 & 2.12 & 4.82 & 30.85 & 0.062 & $<0.001$ \\
\hline HADS-A score $>7$ points & Before & - & - & - & - & - & - & - \\
\hline & After & 0.62 & 1.87 & 1.30 & 2.67 & 11.51 & 0.023 & 0.001 \\
\hline Residence with person at high COVID-19 death risk: yes & Before & -0.18 & 0.84 & 0.48 & 1.45 & 0.413 & 0.001 & 0.520 \\
\hline & After & 0.51 & 1.66 & 1.09 & 2.51 & 5.62 & 0.011 & 0.017 \\
\hline Diabetes mellitus: yes & Before & 1.55 & 4.71 & 1.72 & 12.88 & 9.10 & 0.016 & 0.003 \\
\hline & After & 1.06 & 2.89 & 1.07 & 8.45 & 3.78 & 0.008 & 0.048 \\
\hline Hypertension: yes & Before & 1.05 & 2.86 & 1.15 & 7.11 & 5.13 & 0.009 & 0.024 \\
\hline & After & 0.55 & 1.73 & 0.72 & 4.19 & 1.51 & 0.003 & 0.220 \\
\hline Face washing $>3$ times/day & Before & 0.18 & 1.20 & 0.76 & 1.89 & 0.60 & 0.001 & 0.440 \\
\hline & After & 0.49 & 1.64 & 1.11 & 2.43 & 6.06 & 0.012 & 0.014 \\
\hline Use of soap to wash face: yes & Before & - & - & - & - & - & - & - \\
\hline & After & 0.45 & 1.56 & 1.04 & 2.35 & 4.60 & 0.009 & 0.032 \\
\hline Shower frequency > 3 times/week & Before & 0.42 & 1.52 & 0.97 & 2.38 & 3.30 & 0.006 & 0.069 \\
\hline & After & 0.67 & 1.96 & 1.14 & 3.38 & 5.84 & 0.012 & 0.016 \\
\hline Body xerosis or eczema (redness, dryness, fissures, weepi & ng, crusti & g, burn & g, pain, or & itus on & dy skin) & & & \\
\hline Sex: female & Before & 0.35 & 1.41 & 0.89 & 2.25 & 2.14 & 0.004 & 0.144 \\
\hline & After & 0.86 & 2.36 & 1.59 & 3.48 & 18.47 & 0.036 & $<0.001$ \\
\hline HADS-A score $>7$ points & Before & - & - & - & - & - & - & - \\
\hline & After & 0.47 & 1.61 & 1.13 & 2.30 & 6.79 & 0.014 & 0.009 \\
\hline Shower frequency > 3 times/week & Before & 0.66 & 1.94 & 1.27 & 2.94 & 9.56 & 0.018 & 0.002 \\
\hline & After & 1.20 & 3.32 & 1.84 & 5.97 & 15.99 & 0.035 & $<0.001$ \\
\hline
\end{tabular}


Table 3 | Continued.

\begin{tabular}{|c|c|c|c|c|c|c|c|c|}
\hline \multirow{2}{*}{ Univariate logistic regression analysis } & & \multirow{2}{*}{$\beta \mathbf{i}$} & \multirow{2}{*}{ Odds ratio } & \multicolumn{2}{|c|}{$95 \% \mathrm{Cl}$} & \multirow{2}{*}{ Wald value } & \multirow[b]{2}{*}{$R^{2}$} & \multirow{2}{*}{$p$-value } \\
\hline & & & & Lower & Upper & & & \\
\hline \multicolumn{9}{|l|}{ Acne or folliculitis on face (acne or pimples on face) } \\
\hline \multirow[t]{2}{*}{ Age $\leq 30$ years } & Before & 1.05 & 2.86 & 1.92 & 4.28 & 26.43 & 0.050 & $<0.001$ \\
\hline & After & 1.02 & 2.78 & 1.93 & 4.02 & 29.81 & 0.057 & $<0.001$ \\
\hline \multirow[t]{2}{*}{ Sex: female } & Before & 0.79 & 2.21 & 1.38 & 3.53 & 10.90 & 0.022 & 0.001 \\
\hline & After & 1.12 & 3.08 & 2.01 & 4.70 & 26.98 & 0.055 & $<0.001$ \\
\hline \multirow[t]{2}{*}{ HADS-A score $>7$ points } & Before & - & - & - & - & - & - & - \\
\hline & After & 0.62 & 1.86 & 1.29 & 2.68 & 11.02 & 0.022 & 0.001 \\
\hline \multirow[t]{2}{*}{ Use of soap to wash face: yes } & Before & - & - & - & - & - & - & - \\
\hline & After & 0.41 & 1.61 & 1.10 & 2.32 & 3.71 & 0.010 & 0.047 \\
\hline \multirow[t]{2}{*}{ Use of face masks in hospital: yes } & Before & - & - & - & - & - & - & - \\
\hline & After & 1.62 & 5.05 & 1.14 & 22.32 & 18.88 & 0.013 & 0.033 \\
\hline \multicolumn{9}{|c|}{ Hair loss (any active hair loss complaints, except those in form of round patches) } \\
\hline \multicolumn{9}{|c|}{ Males } \\
\hline \multirow{2}{*}{ Age $\leq 30$ years } & Before & 0.44 & 1.56 & 0.79 & 3.07 & 1.62 & 0.010 & 0.203 \\
\hline & After & 0.74 & 2.10 & 1.04 & 4.25 & 4.27 & 0.027 & 0.039 \\
\hline \multicolumn{9}{|l|}{ Females } \\
\hline \multirow[t]{2}{*}{ Age $\leq 30$ years } & Before & 0.47 & 1.59 & 0.99 & 2.56 & 3.71 & 0.011 & 0.054 \\
\hline & After & 0.20 & 1.23 & 0.77 & 1.95 & 0.73 & 0.002 & 0.394 \\
\hline \multirow[t]{2}{*}{ HADS-A score > 10 points } & Before & - & - & - & - & - & - & - \\
\hline & After & 0.59 & 1.81 & 1.12 & 2.94 & 5.78 & 0.017 & 0.016 \\
\hline Palmar hyperhidrosis (excessive sweating on palms) & & & & & & & & \\
\hline Age $\leq 30$ years & Before & 1.03 & 2.81 & 1.55 & 5.09 & 11.66 & 0.023 & 0.001 \\
\hline & After & 1.07 & 2.92 & 1.77 & 4.81 & 17.61 & 0.034 & $<0.001$ \\
\hline Using gloves: yes & Before & - & - & - & - & - & - & - \\
\hline & After & 1.60 & 4.94 & 1.78 & 20.71 & 4.76 & 0.015 & 0.029 \\
\hline Xeromycteria (drying or bleeding inside nose / nasal muco & sa) & & & & & & & \\
\hline HADS-A score $>7$ points & Before & - & - & - & - & - & - & - \\
\hline & After & 0.47 & 1.60 & 1.05 & 2.43 & 4.90 & 0.010 & 0.027 \\
\hline Nasal washout with any liquid: yes & Before & - & - & - & - & - & - & - \\
\hline & After & 0.58 & 1.79 & 1.11 & 2.89 & 5.61 & 0.011 & 0.018 \\
\hline Acute or chronic urticaria (red, swollen, edematous, itchy, & and fadir & ng lesion & s within $24-\mathrm{hc}$ & ur period & n body) & & & \\
\hline Sex: female & Before & 0.27 & 1.31 & 0.69 & 2.49 & 0.70 & 0.001 & 0.403 \\
\hline & After & 0.78 & 2.17 & 1.27 & 3.72 & 7.92 & 0.017 & 0.005 \\
\hline HADS-A score $>7$ points & Before & - & - & - & - & - & - & - \\
\hline & After & 0.51 & 1.66 & 1.03 & 2.69 & 4.30 & 0.009 & 0.038 \\
\hline Residence with person at high COVID-19 death risk: yes & Before & 0.65 & 1.91 & 1.04 & 3.51 & 4.36 & 0.008 & 0.037 \\
\hline & After & 0.53 & 1.70 & 1.04 & 2.77 & 4.51 & 0.008 & 0.034 \\
\hline Diabetes mellitus: yes & Before & 1.75 & 5.75 & 2.00 & 16.51 & 10.56 & 0.016 & 0.001 \\
\hline & After & 1.51 & 4.52 & 1.65 & 12.36 & 8.65 & 0.015 & 0.003 \\
\hline Aphthous stomatitis (painful, recurrent, superficial or deep & p sores [a & aphthae, & ulcers] in mou & & & & & \\
\hline Residence with person at high COVID-19 death risk: yes & Before & 0.39 & 1.48 & 0.83 & 2.64 & 1.78 & 0.003 & 0.183 \\
\hline & After & 0.53 & 1.70 & 1.04 & 2.77 & 4.51 & 0.008 & 0.034 \\
\hline Diabetes mellitus: yes & Before & 1.48 & 4.40 & 1.54 & 12.53 & 7.69 & 0.012 & 0.006 \\
\hline & After & 1.24 & 3.47 & 1.56 & 9.56 & 5.80 & 0.010 & 0.016 \\
\hline Seborrheic dermatitis (white-yellow scales or dandruff on s & scalp) & & & & & & & \\
\hline Age $\leq 40$ years & Before & 1.16 & 3.18 & 1.49 & 6.79 & 8.92 & 0.022 & 0.003 \\
\hline & After & 0.97 & 2.63 & 1.41 & 4.89 & 9.30 & 0.021 & 0.002 \\
\hline HADS-A score $>12$ points & Before & - & - & - & - & - & - & - \\
\hline & After & 0.82 & 2.27 & 1.16 & 4.45 & 5.65 & 0.011 & 0.017 \\
\hline
\end{tabular}

$\beta i=$ regression coefficient, $\mathrm{Cl}=$ confidence interval, $\mathrm{R}^{2}=\mathrm{Cox} \&$ Snell $\mathrm{R}$ squared, HADS-A = Hospital Anxiety and Depression Scale-Anxiety (0-7 = no anxiety, 8-10 $=$ mild anxiety, 11-14 = moderate anxiety, 12-21 = severe anxiety), PPE = personal protective equipment. + Participants that never used relevant PPE excluded, ¥participants that never used relevant PPE also included in analysis. Significant values are shown in bold.

Table 4 | Correlation of severity of various dermatological complaints with HADS-A scores after COVID-19.

\begin{tabular}{|c|c|c|c|c|}
\hline \multirow{2}{*}{ Control variables } & \multirow{2}{*}{ Complaint-related VAS after COVID-19 } & \multirow{2}{*}{ df } & \multicolumn{2}{|c|}{ Correlation with HADS-A Score } \\
\hline & & & $r$ & $p$-value \\
\hline \multirow[t]{2}{*}{ 1. Sex (male, female)* } & Xerosis cutis on hands & 449 & 0.297 & $<0.001$ \\
\hline & Hand eczema & 359 & 0.292 & $<0.001$ \\
\hline \multirow[t]{10}{*}{ 2. Residence with person at high COVID-19 death risk (yes, no) } & Facial xerosis or eczema & 218 & 0.202 & 0.003 \\
\hline & Body xerosis or eczema & 224 & 0.326 & $<0.001$ \\
\hline & Acne or folliculitis on face & 197 & 0.231 & 0.001 \\
\hline & Hair loss in ment & 41 & 0.297 & 0.053 \\
\hline & Hair loss in woment & 101 & 0.138 & 0.164 \\
\hline & Palmar hyperhidrosis & 69 & 0.415 & $<0.001$ \\
\hline & Xeromycteria & 125 & 0.194 & 0.029 \\
\hline & Acute or chronic urticaria & 84 & 0.228 & 0.035 \\
\hline & Aphthous stomatitis & 92 & 0.238 & 0.021 \\
\hline & Seborrheic dermatitis & 117 & 0.069 & 0.453 \\
\hline
\end{tabular}

VAS = visual analog scale (0 to 10 points = severity of diseases), HADS-A = Hospital Anxiety and Depression Scale-Anxiety (after COVID-19 outbreak), *not used as control variable because hair loss was evaluated separately for both sexes, tparticipants with possible telogen effluvium or androgenic alopecia evaluated by excluding alopecia areata. Spearman's rho partial correlation test was used by syntax. Significant values are shown in bold. 
any complaint in men. However, as is known, hair loss caused by stress may only become apparent after a significant period, and so a more indicative result may be obtained with a follow-up study after a few months.

Sex is known to be a risk factor for some dermatological diseases. Hand eczema, acne, TE, and urticaria are more common in women, and AGA and SD are more common in men (17). Parallel to this knowledge, we found that complaints suggesting xerosis and eczema on the hand, face, and body increased in women before and after the onset of the COVID-19 outbreak. However, the fact that hygiene behavior does not differ by sex except for the frequency of handwashing suggests that genetic factors are also important in these complaints. It was reported in the literature that hygiene behavior does not differ between men and women (18). Unlike AGA, TE seems to be more common in women (19). In this study, male and female volunteers were compared and placed into separate categories, considering that there was a significant difference in diagnosis distribution by sex. However, a differential diagnosis of hair loss could not be made. We found an increase in the frequency of $\mathrm{PH}$ and urticaria in men and an increase in the frequency of all other complaints in women except hair loss. The reason for the absence of a significant increase in the frequency of hair loss in both sexes may be that AGA is a chronic problem and the transition period after the triggering factor for acute shedding is still short.

It was determined that there was an increase in the severity of complaints related to diseases such as only xerosis (not eczema) on the hands, xeromycteria, and SD in men and the severity of complaints related to all other diseases except acne in women. Women are more susceptible to acne, xerosis, and eczema. During the pandemic, hand eczema, body xerosis, and urticaria complaints became more pronounced in women. The fact that most of the symptoms associated with dermatological diseases were more frequent and severe in women was consistent with general expectations $(17,20)$. Age was found to be a risk for acne ( $\leq 30$ years), $\mathrm{PH}$ ( $\leq 30$ years), and SD ( $\leq 40$ years) symptoms. These diseases are seen more frequently in young adults, similar to our findings (17, 21, 22).

Excessive personal hygiene measures can disrupt the hydrolipidic layer of the skin, leading to xerosis and eczema $(8,23)$. It was reported that the risk of hand irritation increased 2.17 times in HCPs washing hands more than 10 times/day during the COVID-19 outbreak (16). In those that wash hands 10 times a day, we found that complaints suggesting xerosis on the hands developed 1.70 times more likely before the COVID-19 outbreak and 2.44 times more likely during the outbreak. The risk of hand eczema is positively associated with the frequency of handwashing. We found that $\geq 10$ seconds of handwashing time increased the risk of eczema 5.44 times during the COVID-19 outbreak. In parallel, the need for moisturizers increased approximately five times in participants with complaints suggesting hand xerosis and 1.6 times in those with complaints suggesting hand eczema during the COVID-19 outbreak. We found that washing the face more than three times a day and using soap when washing the face increased the risk of facial xerosis or eczema by approximately 1.5 times during the COVID-19 outbreak. It was found that taking a shower more than three times a week increased the risk of body xerosis or eczema 1.94 times before the pandemic and 3.32 times during the pandemic period. Although there was no association between the frequency of face washing and acne, washing the face with soap increased the risk of developing acne by 1.61 times during the pandemic period. Kairavee et al. concluded that washing the face with soap is an acne trigger (24). Many studies have reported that washing the face more than three times a day can exacerbate acne with a rebound effect due to irritation of the sebaceous glands (25, 26). We also found that the xeromycteria risk increased 1.79 times in those that engaged in nasal irrigation with any liquid.

In our study, the risk of developing symptoms related to hand xerosis during the COVID-19 outbreak was found to increase by 2.68 times overall in people that used gloves and 2.21 times in those that used gloves for at least 2 hours. Using gloves for at least 1 hour increased the risk of hand eczema by 1.78 times. Foo et al. reported that long-term use of gloves increased xerosis and dermatitis on the hands (27). However, our study showed that the risk also increased considerably with short-term glove use (for 1 to 2 hours). In the literature, more than a third of HCPs are reported to have experienced various skin reactions, including acne, itching, and dermatitis, caused by the use of $\operatorname{N95}$ masks $(8,27)$. However, we found no relationship between xerosis or eczema and mask use. On the other hand, it was remarkable that the risk of acne increased fivefold as a result of the use of any mask during the COVID-19 outbreak. Foo et al. reported that acne lesions were observed in $59.6 \%$ of HCPs using N95 masks for more than 8 hours a day (27). However, we found no relationship between mask-wearing time and acne formation. Possible mechanisms for mask-induced acne pathogenesis include friction or bursting of comedones, occlusion of the pilosebaceous duct, and formation of a wet environment conducive to bacterial proliferation (23). In our study, it was determined that the risk of $\mathrm{PH}$ increased by 4.94 times with glove use, but $\mathrm{PH}$ was not related to the duration of use among those wearing gloves. SD exacerbation was reported on the scalp and face resulting from the use of a hair bonnet and mask (23). However, we found no relationship between mask use and SD. Because it would have created complexity, facial symptoms suggestive of SD were not questioned in this study. These symptoms were evaluated under the category of facial eczema. The use of PPE is claimed to increase the colonization of bacteria in the oral mucosa and may also cause the development of oral mucosal diseases such as stomatitis and oral ulcers (23). However, the findings of our study did not support this knowledge. Among the participants questioned about the presence of pressure rash or erosion around the nose, forehead, eyes, and ears, $28 \%$ developed involvement in at least one area, most commonly on the nasal bridge (15.9\%), consistent with reports by Lan et al. (16).

Increasing cases, high workload, PPE depletion, and the possibility of infecting the family may raise the mental burden of HCPs during the pandemic. In a recent study in China, psychological disorders such as depression, anxiety, and insomnia have occurred in some HCPs, especially women and nurses, during the COVID-19 outbreak (28). In our study, anxiety levels were higher in women. However, among those that resided with people at high risk for COVID-19, anxiety levels were higher in men. Although the HADS-A scores were significantly higher in nurses as a femaleweighted group, there was no difference between doctors and nurses in terms of anxiety levels when sex was adjusted. As seen in Table 1, hand washing and showering frequencies show a weak but significant correlation with anxiety levels. Indeed, it was determined that the frequency of symptoms related to xerosis and/ or eczema on the hands, face, and body significantly increased among the participants that had at least mild anxiety levels (HADS-A > 7 points). The highest risk increase was in complaints suggesting xerosis (about six times) and hand eczema (about 2.5 
times). Residence with a person at high risk of death from COVID-19 was found to increase the risk from 1.66 to 2.34 times in complaints related to xerosis and eczema. Participants with at least mild anxiety levels ( $>7$ points) were found to have at least 1.5 to two times increased risk of complaints suggesting acne, xeromycteria, and urticaria. It has been found that the frequency of hair loss in women with at least moderate anxiety levels ( $>10$ points) increased by 1.81 times, and the risk of complaints suggesting SD increased by 2.27 times in those with severe anxiety levels (> 12 points). Stress is a well-known risk factor in diseases such as acne, hair loss, urticaria, and SD (29-31). HADS-A scores have not been found to be associated with the frequencies of PH and AS. Correlations between the severity of the complaints and anxiety levels were investigated, and the severity of all complaints except hair loss and SD was significantly correlated with anxiety level.

Our study has some limitations. First of all, this is a self-administered questionnaire study. Therefore, the questions asked about the symptoms give an idea about the diagnosis, but it is difficult to make a definitive diagnosis with this method. Other dermatological diseases with the possibility of exacerbation (atopic dermatitis and rosacea, etc.) could not be asked about in order not to cause conflicting discernments. Our study was conducted on a group familiar with medical terminology. The groups were not identical in terms of sex, and no comparison was made between professions. However, before analysis of the frequency or severity of symptoms, female nurses and doctors were compared informally and consistent results were obtained.

In conclusion, our study showed that the frequency and severity of some dermatological complaints, particularly related to xerosis and eczema, increased in HCPs due to PPE use, increased hygiene behavior, and anxiety. Especially women are at higher risk for dermatological complaints during the pandemic. We think that these complaints can be reduced with the use of PPE at optimal times, reasonable hygiene, basic protective measures, and psychosocial support to HCPs, and that these might have positive implications for some dermatological complaints.

\section{References}

1. Guo YR, Cao QD, Hong ZS, Tan YY, Chen SD, Jin HJ, et al. The origin, transmission and clinical therapies on coronavirus disease 2019 (COVID-19) outbreak-an update on the status. Mil Med Res. 2020;7:11.

2. Singhal T. A review of coronavirus disease-2019 (COVID-19). Indian J Pediatr. 2020;87:281-6.

3. Rothe C, Schunk M, Sothmann P, Bretzel G, Froeschl G, Wallrauch C, et al. Transmission of 2019-nCoV infection from an asymptomatic contact in Germany. N Engl J Med. 2020;382:970-1.

4. Cheng ZJ, Shan J. 2019 Novel coronavirus: where we are and what we know. Infection. 2020;48:155-63.

5. Guan WJ, Ni ZY, Hu Y, Liang WH, Ou CQ, He JX, et al. Clinical characteristics of coronavirus disease 2019 in China. N Engl J Med. 2020;382:1708-20.

6. Gautier JF, Ravussin Y. A new symptom of COVID-19: loss of taste and smell. Obesity. 2020;28:848.

7. Remuzzi A, Remuzzi G. COVID-19 and Italy: what next? Lancet. 2020;395:12258.

8. Darlenski R, Tsankov N. Covid-19 pandemic and the skin: what should dermatologists know? Clin Dermatol. 2020. [Epub ahead of print].

9. Aydemir Ö, Guvenir T, Kuey L, Kultur S. [Validity and reliability of Turkish version of the Hospital Anxiety and Depression Scale]. Turk Psikiyatri Derg. 1997;8:2807. Turkish.

10. Julian LJ. Measures of anxiety: State-Trait Anxiety Inventory (STAI), Beck Anxiety Inventory (BAI), and Hospital Anxiety and Depression Scale-Anxiety (HADS-A). Arthritis Care Res (Hoboken). 2011;63:S467-72.

11. World Health Organization. Infection prevention and control during health care when COVID-19 is suspected: interim guidance [Internet]. Geneva. 2020 [cited 2020 May 04]. Available from: https://www.who.int/publications-detail/ infection-prevention-and-control-during-health-care-when-novel-coronavirus(ncov)-infection-is-suspected-20200125/.

12. Center for Disease Control and Prevention. Infection control guidance for healthcare professionals about coronavirus (COVID-19). Atlanta. 2020 [cited 2020 May 04]. Available from: https://www.cdc.gov/coronavirus/2019-ncov/hcp/infection-control.html/.

13. Kantor J. Behavioral considerations and impact on personal protective equipment use: Early lessons from the coronavirus (COVID-19) pandemic. J Am Acad Dermatol. 2020;82:1087.

14. Flyvholm MA, Bach B, Rose M, Jepsen KF. Self-reported hand eczema in a hospital population. Contact Dermatitis. 2007;57:110-5.

15. Hamnerius N, Svedman C, Bergendorff O, Björk J, Bruze M, Pontén A. Wet work exposure and hand eczema among healthcare workers: a cross-sectional study. Br J Dermatol. 2018;178:452-61.
16. Lan J, Song Z, Miao X, Li H, Li Y, Dong L, et al. Skin damage among health care workers managing coronavirus disease-2019. J Am Acad Dermatol. 2020;82: $1215-6$.

17. Bilgili ME, Yildiz H, Sarici G. Prevalence of skin diseases in a dermatology outpatient clinic in Turkey. A cross-sectional, retrospective study. J Dermatol Case Rep. 2013;7:108.

18. Campos MA, Sousa AC, Varela P, Baptista A. Evaluation of hygiene habits: crosssectional study. Acta Dermatovenerol Alp Pannonica Adriat. 2016;25:45-8.

19. Burroway B, Griggs J, Martinez-Velasco MA, Tosti A. Hair and scalp treatments. Cham (Switzerland): Springer; c2020. Chapter 10, Telogen Effluvium; p. 125-38.

20. Shrestha P, Mikrani JA. Pattern of dermatological disease and its relation to gender in Lumbini Medical College Teaching Hospital. J Lumbini Med Coll. 2015;3: $16-8$.

21. Zeichner JA, Baldwin HE, Cook-Bolden FE, Eichenfield LF, Fallon-Friedlander S, Rodriguez DA. Emerging issues in adult female acne. J Clin Aesthet Dermatol. 2017;10:37-46.

22. Romero FR, Haddad GR, Miot HA, Cataneo DC. Palmar hyperhidrosis: clinical, pathophysiological, diagnostic and therapeutic aspects. An Bras Dermatol. 2016;91:716-25.

23. Yan Y, Chen H, Chen L, Cheng B, Diao P, Dong L, et al. Consensus of Chinese experts on protection of skin and mucous membrane barrier for health-care workers fighting against coronavirus disease 2019. Dermatol Ther. 2020:e13310.

24. Kairavee D, Vivek C. Factors aggravating or precipating acne. Natl J Commun Med. 2010;1:4-6.

25. Hastuti R, Mustifah EF, Alya I, Risman M, Mawardi P. The effect of face washing frequency on acne vulgaris patients. JDVI. 2019:35-40.

26. Kubba R, Kumar B, Thappa DM, Sharma R, Vedamurthy M, Dhar S, et al. Cosmetics and skin care in acne. Indian J Dermatol Venereol Leprol. 2009;75:55-6.

27. Foo $\mathrm{CCl}$, Goon ATJ, Leow YH, Goh CL. Adverse skin reactions to personal protective equipment against severe acute respiratory syndrome-a descriptive study in Singapore. Contact Dermatitis. 2006;55:291-4.

28. Lai J, Ma S, Wang Y, Cai Z, Hu J, Wei N, et al. Factors associated with mental health outcomes among health care workers exposed to coronavirus disease 2019. JAMA Netw Open. 2020;3:e203976.

29. Jović A, Marinović B, Kostović K, Čeović R, Basta-Juzbašić A, Bukvić Mokos Z. The impact of psychological stress on acne. Acta Dermatovenerol Croat. 2017; 25:133-41.

30. Misery L, Touboul S, Vinçot C, Dutray S, Rolland-Jacob G, Consoli SG, et al. Stress and seborrheic dermatitis. Ann Dermatol Venereol. 2007;134:833-7.

31. Vargas EL, Peña MP, Vargas AM. Influence of anxiety in diverse cutaneous diseases. Actas Dermosifiliogr. 2006;97:637-43. 